\title{
Application of Liposomes Nanoparticles in the Treatment of Malaria: A Mini Review
}

\author{
Farouk S Nas ${ }^{1}$, Yahaya $\mathrm{A}^{2}$ and Muhammad $\mathrm{Ali}^{3 *}$ \\ ${ }^{1}$ Department of Biological Science, Bayero University Kano, Nigeria \\ ${ }^{2}$ Department of Biological Science, Kano University of Science and Technology, Nigeria \\ ${ }^{3}$ Department of Biological Science, Federal University Gusau, Nigeria \\ ${ }^{*}$ Corresponding author: Muhammad Ali, Department of Biological Science, Federal University Gusau, Nigeria
}

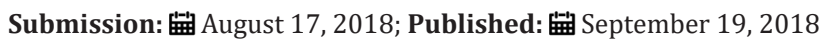

\begin{abstract}
Malaria is considered as complicated disease due to its variation in epidemiology and clinical symptoms in different part of the world. The Plasmodium parasite was found to be resistance to several antimalarial drugs over a period of time and such drug resistance has emerged as one of the greatest challenges facing the control of malaria today. One possible solution to the problems associated with anti-malarial pharmaceutical is the use of nanomaterials such as liposomes. Liposomes improve the therapeutic efficacy by enhancing drug absorption while avoiding or minimizing rapid degradation and side effects, prolonging the biological half-life and reducing toxicity. The unique feature of liposomes is that they are biocompatible and biodegradable lipids and are inert and non-immunogenic. Liposomes can compartmentalize and solubilize both hydrophilic and hydrophobic materials. All these properties of liposomes and their flexibility for surface modification to add targeting moieties make liposomes more attractive candidates for use as effective drug delivery vehicles which could have potential for malaria eradication. This paper reviews the application of liposomes in the treatment of Plasmodium parasite.
\end{abstract}

Keywords: Liposomes; Malaria; Nano; Nanotechnology; Plasmodium; Phospholipid

\section{Introduction}

Malaria remains as one of the most important disease of public health concern in countries where transmission of the disease occurs regularly [1]. Malaria is considered as complicated disease due to its variation in epidemiology and clinical symptoms in different part of the world. Five species of the genus Plasmodium are found to affect human. According to Olliaro [2], the 5 species include $P$. falciparum, $P$. malariae, $P$. vivax, $P$. ovale and $P$. knowlesi. Malaria is a vector borne disease in which the Plasmodium parasites are transmitted to human through the bite of infected female anopheles' mosquito [3]. The malaria continues to be a major threat to the world. In 2011, there is an estimation of more than 3.3 billion people who are at risk of malaria and most of them are associated to sub Saharan Africa [4]. According to World Health Organization (WHO), there are over 200 million cases of malaria annually in which $80 \%$ of the cases and about $90 \%$ of malaria death cases were estimated to occur in African region. The report concluded that children of less than 5 years of age and pregnant women are affected most [5]. The Plasmodium parasite was found to be resistance to several antimalarian drugs over a period of time and such drug resistance has emerged as one of the greatest challenges facing the control of malaria today [2].

Additionally, contributing to the high incidence of infection, malaria parasites are becoming resistant to the current proposed drugs (chloroquine, pyrimethamine, aretesunate, sulfadoxine, etc.). Therefore, the development of a new and effective antimalarial agent is needed. The World Health Organization has recommended a combination of anti-malarials and aretesunate to treat uncomplicated malaria cases. An endemic is possible if malaria builds resistance to these drug combinations [4-6]. One possible solution to the problems associated with anti-malarial pharmaceutical is the use of nanomaterials. The use of colloidal drug carriers (liposomes and micro/nanoparticles) provide versatility in site specific or targeted drug delivery along with controlled optimal drug release [7,8]. Nanoparticles have added advantages over micro-particles such as bioavailability, the ability to improve drug encapsulation, pharmacokinetics, and therapeutic therapy [9].

Nanotechnology is the study of extremely small structures. The prefix 'nano' refers to a very small or miniature size [10]. Using nanotechnology, individual atoms, molecules or compound can be treated into structure to produce materials and devices with special properties [10]. Nanotechnology is being applied extensively to provide targeted drug therapy, diagnostics, tissue regeneration, cell culture, biosensors and other tools in the field of molecular biology [10]. Through nanotechnology, various flat forms like nanotubes, liposomes, fullers, nanopores, quantum dots, magnetic nanoprobes and radio-controlled nanoparticles are being 
developed [10]. Nanomaterials may be strategically advantageous as active antibacterial groups since their surface area is exceedingly large relative to their size. Nanosized particles are very effective and may provide high activity although only a small dose of the particles is used [11]. Liposomes are microscopic structures made up of one or more concentric spheres of lipid bilayers enclosing aqueous compartments which are extensively used for controlled delivery drug formulations [11].

\section{Liposomes}

Liposomes are small and artificial spherical shape vesicles that can be created from natural non-toxic phospholipids and cholesterol. Due to their size and hydrophilic and hydrophobic characters in addition to biocompatibility, the liposomes are considered as promising systems for drugs delivery [12]. Liposomes properties differ considerably with surface charge, lipid composition, sizes and general method of preparation [13] Furthermore, the choice of bilayer components determined the 'fluidity' or 'rigidity' and the charge of the bilayer [14]. For example, unsaturated phosphatidylcholine species from natural sources such as egg or soybeans give much more permeable and less stable bilayers, whereas the saturated phospholipid with long acyl chains (for example, dipalmitoylphos phatidylcholine) form a rigid, rather impermeable bilayer structure [12-15]. It has been displayed that phospholipids impulsively form closed structures when they are hydrated in aqueous solutions. Such vesicles which have one or more phospholipid bilayer membranes can transport lipid or aqueous drugs, depending on the nature of the drugs [16]. Because lipids are amphipathic (both hydrophobic and hydrophilic) in aqueous media, their thermodynamic phase properties and self-assembling characteristics influence entropically focused confiscation of their hydrophobic sections into spherical bilayers. Those layers are called lamellae [16].

Generally, liposomes are definite as spherical vesicles with particle sizes ranging from $30 \mathrm{~nm}$ to several micrometers. They consist of one or more lipid bilayers surrounding aqueous units, where the polar head groups are oriented in the pathway of the interior and exterior aqueous phases [17]. Liposomes are extensively used as carriers for numerous molecules in pharmaceutical and cosmetic industries. Additionally, food and farming industries have extensively studied the uses of liposomes encapsulation to grow delivery system that can entrap unstable compound such as antioxidant, antimicrobials, flavors and bioactive elements and as well shield their functionality [17]. Liposomes can trap both hydrophilic and hydrophobic compound, avoid decomposition of the entrapped combinations, and release the entrapped at designated targets [18-20]. Because of their biodegradability, low toxicity biocompatibility and aptitude to trap both hydrophilic and lipophilic drugs [21] and simplify site specific drug delivery to tumor tissue [22], liposomes have increased rate both as an investigational system and commercially as a drug-delivery system (Figure 1). Many studies have been conducted on liposomes with the aim decreasing drug toxicity and targeting specific cells [23-25].

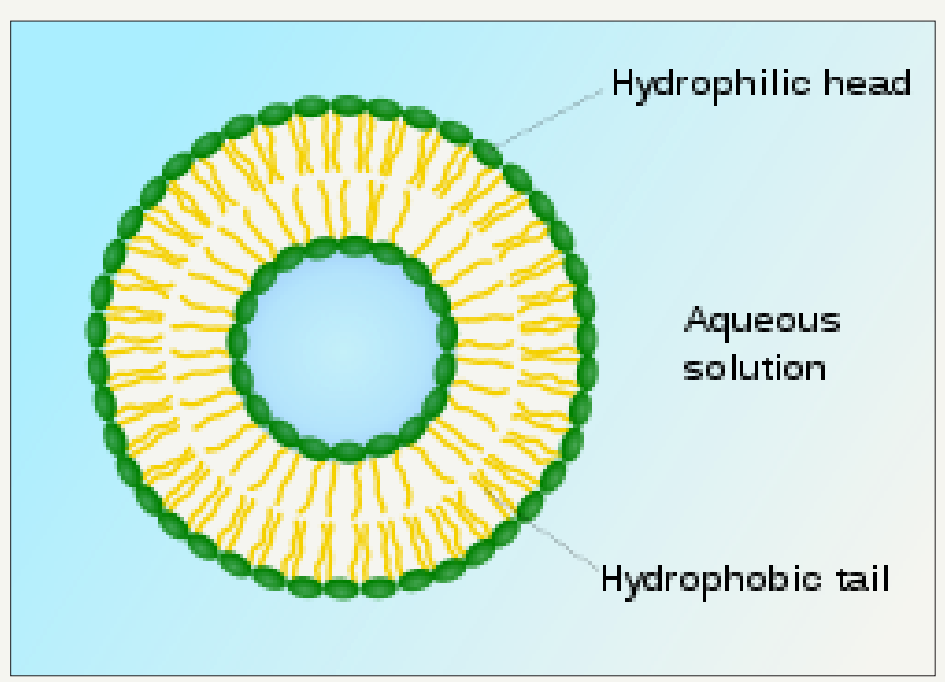

Figure 1: Structure of liposomes [26].

\section{Liposomes as nanocarrier for antimalarial drugs}

Liposomes are synthetic structures that consisted of several hundred nanometers in diameter containing one or several phospholipid bilayers enclosing an aqueous core [26,27]. The concept of utilizing liposomes as vehicle for drug delivery system was introduce in 1970s and more recently the use of liposomes nanocarriers has been extended to immunological adjuvants and as delivery vehicles for vaccine especially to specific target cells [28]. Both lipophilic and hydrophilic particles can be incorporated into liposomes and delivered to target sites within the host organism.
Hydrophilic particles including peptides, proteins and nucleic acid can be entrapped within the inner aqueous phase while lipophilic drugs such as adjuvants and lipopeptides can be incorporated onto the outer phospholipid layer. Liposomes are immunologically advantageous due to their targeting and uptake by professional antigen presenting cells, and additionally antigens, adjuvants and antibodies can be attached to the outer surface of liposomes to facilitate delivery into infected cells [28]. Optimal combinations of antigens, antibodies and adjuvants give liposomes plasticity and allow the opportunity for optimization of different drug regimens. 
Liposomes have shown significant effect as nanocarrier for the prophylaxis and also for the treatment of malaria and as well as for vaccine delivery for the prevention of malaria [29]. Currently, effective therapy for malaria is limited due to toxic drug side effects and the development of resistance to current drug regimens. Encapsulation of therapeutic agents within liposomes can favorably alter the dose and distribution of drugs within the body, which may significantly reduce unwanted toxic side effects, increase treatment efficacy and reduce the risk of drug resistance [30]. Presently, malaria vaccine strategies suffer from the problem of resistance to recombinant antigens and as well the need for frequent re-boosting. The use of live-attenuated parasites is limited mainly because high doses of Plasmodium are needed and because a clinically appropriate route for inoculation has not been found [31]. Liposomes have advantage over other vaccine delivery system the carrier vesicles protect its contents from degradation within the host are biocompatible, non - toxic and selective. Novel gel core liposomes which uses a combination of polymer and lipid delivery systems have been recently developed and tested for the controlled delivery of malarial antigen Pfs 25 combined with CpGODN, a potent immunostimulatory vaccine adjuvant [32]. Gel core liposomes increase liposome stability by incorporation of a polymer into the internal aqueous phase of the liposome that allows for slower drug delivery. The rate of release is controlled by slow diffusion through the polymer gel and through the phospholipid bilayers which enable manipulations of drug concentration within liposome vehicle to enable the ability of long-term antigen persistence which could decrease the need for boosting. Additionally, novel RTS, S-based vaccine formulations that utilize a liposome-based adjutant are currently undergoing clinical trial [31].

The RTS.S/AS01B vaccine induces high antibody responses and at the same time improves $\mathrm{T}$ cell responses to the Circumsporozoite protein (CSP) in mice and in non-human primates [33]. Antimalaria drugs showed different degree of toxicity, which limit their use. Current therapeutic administration strategies release free drugs into the blood and offer little specificity regarding infected cells [33]. Early studies have indicated that the liposomalization of the antimalarial agent chloroquine increases its maximal tolerable dose and its efficiency and activity against murine malarial infections greater than just chloroquine alone [34,35]. Moreover, the ability to increase the doses of chloroquine per injection after liposome encapsulation allowed successful treatment of infection with chloroquine resistant $P$. berghei which could be cured by a seven-day course with the maximum tolerable dose of free chloroquine [35]. More recently, antibody coated liposomes loaded with antimalarial drugs such as primaquine and chloroquine completely arrested human infecting parasite, $P$. falciparum growth in vitro and cleared infections [36]. This success was attributed to dual therapeutic and prophylactic effect achieved with the use of liposome vesicles targets to both infected and non-infected erythrocytes [36]. Resistance to current antimalarial therapy is attributed to a large genetic diversity of Plasmodium strain, specific mutation in $P$. falciparum chloroquine transporter gene and multi drug resistance genes in P. falciparum [37].
Liposomes circumvent drug resistant malaria because they are targeted for intracellular delivery which bypasses chloroquine transponder and pass through cell membrane by alternative mechanisms such as membrane fusion or entrapment of chloroquine in $\mathrm{pH}$-sensitive liposomes [38]. Directing liposomes to parasite-infected erythrocytes is another strategy that would allow for selective drug distribution and allow for exposure of lethal doses directly to the pathogens [38]. Ligands conjugated to the surface of liposomes can be used to target and specifically bind Plasmodium infected cell [39]. Because the blood-stage of Plasmodium infection is responsible for all symptoms and pathologies of malaria, Plasmodium -infected erythrocytes are the main antimalarial therapeutic target. The targeting of liposomes to erythrocytes using heparin and monoclonal antibodies to erythrocyte surface proteins have been studied in vitro and have shown promise towards targeted drug delivery. Marques et al. [39] encapsulated primaquine in heparin-coated liposomes, this formulation was demonstrated to have antimalarial activity and specific binding affinity for Plasmodium-infected erythrocytes in vitro via heparin targeting of heparin-binding proteins in erythrocyte membranes. Antibody-mediated erythrocytes targeting using liposomes are another promising strategy for targeted drug release. Recently, drugs carried by liposomes were shown to be specifically targeted in vitro to $P$. falciparum infected erythrocytes relative to noninfected erythrocytes likely by docking to infected cell surfaces to facilitate membrane fusion [40]. This demonstrates the feasibility of constructing a carrier able to completely discriminate infected from non-infected erythrocytes.

The fast, specific targeting of antibody labeled liposomes towards Plasmodium- infected cells can facilitate adjusting the amount of encapsulated drugs to a low overall concentration that however guarantees a localized delivery of highly toxic doses only to infected cells. This, in turn, opens perspectives for the use in antimalarial therapy of already existing drugs that are not being tested because of their high toxicity and/or elevated specificity. Recently, liposomes have also been targeted toward hepatocyte to determine their ability to combat liver parasite in murine model of $P$. berghei infection [41]. In this study, the targeting of liposomes to the liver was achieved by expressing a 19 amino acid sequence of a protein expressed by the $P$. berghei circumsporozoite which was chemically bound to the surface of PEGylated liposomes. Peptide targeted liposomes were 100 times more selective to hepatocytes than to cells of other organs which present a great approach for targeting antimalarial drugs to the liver. Targeting antimalarial drugs to Plasmodium infected erythrocytes and hepatocytes using liposomes reduces toxicity, improves therapeutic efficiency, and prolongs drug release compared to conventional approaches [42].

In a study conducted by Rajendran et al. [43] on Therapeutic efficacy of chloroquine in long circulating liposome formulations against chloroquine-resistant Plasmodium berghei infection in mice, the result showed that Chloroquine (CQ) in long circulating liposome formulations with 5mol\% DSPE-mPEG 2000 resulted in enhanced killing of blood parasites compared with similar dose of free chloroquine. Compared with free drug, liposomal formulations 
showed enhanced efficacy assessed by clearance of parasitemia and significant delay in death of mice with Plasmodium berghei CQresistant infections.

\section{Challenges of liposomes assisted drug delivery for ma- laria}

No drug delivery system is faultless; this is the case with liposomes as well. As liposomes are used to enhance and to increase the efficacy of a drug, the cost as well as all the other implications thereof must be taken into account. Cost is an issue when it comes to lipid drug delivery systems, as these systems are quite expensive to produce. The cost is high because of high costs associated with the raw materials used in lipid excipients as well as expensive equipment needed to increase manufacturing [44]. In most cases liposomal formulations are nontoxic, but certain formulations such as the cationic formulations tend to be cytotoxic. This is especially true when liposomal doses are very high [45]. The sterilisation of liposomes is a complicated conundrum, as liposomes are sensitive to high temperatures, as well as certain methods of radiation. Sterilising with chemicals is not a viable option either, as it may affect the stability of the liposomes. The only method for creating sterile liposomes is by filtering the liposomes through a $0.22 \mu \mathrm{m}$ membrane filter after production. This method is only suitable if the liposomes are smaller than $0.2 \mu \mathrm{m}$ in diameter. This method does not remove viruses [46]. Another option is filtering the initial solutions through $0.45 \mu \mathrm{m}$ regenerated cellulose filters and glass fibre filters before starting production, thereafter the entire production process must be done under aseptic conditions [47]. For a pharmaceutical product to be viable for the market, it requires the product to be stable in some form or another for at least a year and a half to two years. To achieve this with liposomes is very difficult if the liposomes remain in suspension. Other methods may be used to increase the shelf life of liposomes, such as freeze-drying after production. Two factors play a major role in the stability of liposomes namely, chemical and physical degradation. The chemical degradation of liposomes is attributed to oxidation and hydrolysis. Physical degradation is most often attributed to the difference in the packing density of the lipids in the bilayer structure. Physical degradation is also a huge factor when formulations are freezedried. When products are freeze-dried a so called cryoprotector must be added to ensure the product is stable when reconstituted.

\section{Conclusion}

In recent years, nanoparticles such as liposomes have attracted a considerable attention as potential carriers for the controlled and site-specific delivery of drugs. The nanodrug delivery systems seem to be a promising and viable approach for improving malaria treatment. Thus liposomal approach can be successfully utilized to improve the pharmacokinetics and therapeutic efficacy simultaneously reducing the toxicity of various highly potent drugs.

\section{References}

1. World Health Organization (WHO) (2011). Guidelines for the treatment of malaria. $\left(2^{\text {nd }}\right.$ edn), Rev. 1. World Health Organization, Geneva, Switzerland.
2. Olliaro P (2005) Drug resistance hampers our capacity to roll back malaria. Clin Infect Dis 41(Supp14): S247-S257.

3. Ridley RG (2005) Malaria: To kill a parasite. Nature 424(6951): 887-889

4. World Health Organization (WHO) (2014) WHO status report on Artemisinin resistance: September. WHO Press, Geneva, Switzerland.

5. World Health Organization (WHO) (2012) World malaria report. World Health Organization, Geneva, Switzerland.

6. Klein EY (2013) Antimalarial drug resistance: A review of the biology and strategies to delay emergence and spread. Int J Antimicrob Agents 41(4): 311-317.

7. Petersen I, Eastman R, Lanzer M (2011) Drug-resistant malaria: Molecular mechanisms and implications for public health. FEBS Lett 585(11): 1551-1562.

8. Trape JF (2001) The public health impact of chloroquine resistance in Africa. Am J Trop Med Hyg 64(1-2 Suppl): 12-17.

9. Roper C, Pearce R, Bredenkamp B, Gumede J, Drakeley C, et al. (2003) Antifolate antimalarial resistance in Southeast Africa: A populationbased analysis. Lancet 361(9364): 1174-1181.

10. Surendiran A, Sandhiya S, Pradhan SC, Adithan C (2009) Novel applications of nanotechnology in medicine. Indian J Med Res 130(6): 689-701.

11. Bangham AD, Standish MM, Watkins JC (1965) Diffusion of univalent ions across the lamellae of swollen phospholipids. J Mol Biol 13(1): 238252.

12. Sahoo SK, Labhasetwar V (2003) Nanotech approaches to drug delivery and imaging. Drug Discov Today 8(24): 1112-1120.

13. Akbarzadeh A, Rezaei SR, Davaran S, Joo SW, Zarghami N, et al. (2013) Liposome: classification, preparation and applications. Nanoscale Res Lett 8(1): 102.

14. Gabizon A, Goren D, Cohen R, Barenholz Y (1998) Development of liposomal anthracyclines: From basics to clinical applications. J Control Release 53(1-3): 275-279.

15. Allen TM (1997) Liposomes. Opportunities in drug delivery. Drugs 54(Suppl 4): 8-14.

16. Chrai SS, Murari R, Imran A (2001) Liposomes: A review. Bio Pharm 14(11): 10-14.

17. Andreas W, Karola VU (2011) Liposome technology for industrial purposes. J Drug Delivery 2011: 9.

18. Atrooz OM (2011) Effects of alkylresorcinolic lipids obtained from acetonic extract of Jordanian wheat grains on liposome properties. Int J Biol Chem 5(5): 314-321.

19. Benech RO, Kheadr EE, Laridi R, Lacroix C, Fliss I (2002) Inhibition of Listeria innocua in cheddar cheese by addition of nisin Z in liposomes or by in situ production in mixed culture. Appl Environ Microbiol 68(8): 3683-3690.

20. Shehata T, Ogawara K, Higaki K, Kimura T (2008) Prolongation of residence time of liposome by surface-modification with mixture of hydrophilic polymers. Int J Pharm 359(1-2): 272-279.

21. Johnston MJ, Semple SC, Klimuk SK, Ansell S, Maurer N, et al. (2007) Characterization of the drug retention and pharmacokinetic properties of liposomal nanoparticles containing dihydrosphingomyelin. Biochim Biophys Acta 1768(5): 1121-1127.

22. Hofheinz RD, Gnad Vogt SU, Beyer U, Hochhaus A (2005) Liposomal encapsulated anti-cancer drugs. Anticancer Drugs 16(7): 691-707.

23. Stano P, Bufali S, Pisano C, Bucci F, Barbarino M, et al. (2004) Novel camptothecin analogue (gimatecan)-containing liposomes prepared by the ethanol injection method. J Liposome Res 14(1-2): 87-109. 
24. Schiffelers RM, Storm G, Bakker Woudenberg IA (2001) Host factors influencing the preferential localization of sterically stabilized liposomes in Klebsiella pneumoniae-infected rat lung tissue. Pharm Res 18(6): 780-787.

25. Omri A, Suntres ZE, Shek PN (2002) Enhanced activity of liposomal polymyxin B against Pseudomonas aeruginosa in a rat model of lung infection. Biochem Pharmacol 64(9): 1407-1413.

26. https://www.wikipedia.org/

27. Santos Magalhães NS, Mosqueira VC (2010) Nanotechnology applied to the treatment of malaria. Adv Drug Deliv Rev 62(4-5): 560-575.

28. Lasic DD (1998) Novel applications of liposomes. Trends Biotechnol 16(7): 307-321.

29. Salvador A, Hernández RM, Pedraz JL, Igartua M (2012) Plasmodium falciparum malaria vaccines: current status, pitfalls and future directions. Expert Rev Vaccines 11(9): 1071-1086.

30. Tiwari S, Goyal AK, Mishra N, Khatri K, Vaidya B, et al. (2009) Development and characterization of novel carrier gel core liposomesbased transmission blocking malaria vaccine. J Control Release 140(2): 157-165.

31. Mettens P, Dubois PM, Demoitié MA, Bayat B, Donner MN, et al. (2008) Improved $\mathrm{T}$ cell responses to Plasmodium falciparum circumsporozoite protein in mice and monkeys induced by a novel formulation of RTS, S vaccine antigen. Vaccine 26(8): 1072-1082.

32. Agrawal AK, Singhal A, Gupta CM (1987) Functional drug targeting to erythrocytes in vivo using antibody bearing liposomes as drug vehicles. Biochem Biophys Res Commun 148(1): 357-361.

33. Owais M, Varshney GC, Choudhury A, Chandra S, Gupta CM (1995) Chloroquine encapsulated in malaria-infected erythrocyte-specific antibody-bearing liposomes effectively controls chloroquine- resistant Plasmodium berghei infections in mice. Antimicrob Agents Chemother 39(1): 180-184.

34. Peeters PA, Huiskamp CW, Eling WM, Crommelin DJ (1989) Chloroquine containing liposomes in the chemotherapy of murine malaria. Parasitology 98(Pt 3): 381-386.

35. Moles E, Urbán P, Jiménez DMB, Viera MS, Angulo BI, et al. (2015) Immunoliposome-mediated drug delivery to Plasmodium-infected and non-infected red blood cells as a dual therapeutic/prophylactic antimalarial strategy. J Control Release 210: 217-229.

36. Biagini GA, Fidock DA, Bray PG, Ward SA (2005) Mutations conferring drug resistance in malaria parasite drug transporters Pgh1 and PfCRT do not affect steady-state vacuolar $\mathrm{Ca}_{2}{ }^{+}$. Antimicrob Agents Chemother 49(11): 4807-4808.

37. Qiu L, Jing N, Jin Y (2008) Preparation and in vitro evaluation of liposomal chloroquine diphosphate loaded by a transmembrane $\mathrm{pH}$ gradient method. Int J Pharm 361(1-2): 56-63.

38. Sailaja AP, Amareshwar P, Chakravarty P (2011) Different techniques used for the preparation of nanoparticles using natural polymers and their application. International Journal of Pharmacy and Pharmaceutical Science 3(2): 45-50.

39. Urbán P, Estelrich J, Cortés A, Fernàndez Busquets X (2011) A nanovector with complete discrimination for targeted delivery to Plasmodium falciparum-infected versus non-infected red blood cells in vitro. J Control Release 151(2): 202-211.

40. Longmuir KJ, Robertson RT, Haynes SM, Baratta JL, Waring AJ (2006) Effective targeting of liposomes to liver and hepatocytes in vivo by incorporation of a Plasmodium amino acid sequence. Pharm Res 23(4): 759-769.

41. Dennis E, Peoples VA, Johnson F, Bibbs RK, Topps D, et al. (2015) Utilizing Nanotechnology to Combat Malaria. J Infect Dis Ther 3: 229.

42. Rajendran V, Hasan GM, Kumar N, Dutt S, Garg N, et al. (2016) Therapeutic efficacy of chloroquine in long circulating liposome formulations against chloroquine-resistant Plasmodium berghei infection in mice. European Journal of Biomedical and Pharmaceutical sciences 3(11): 258-264.

43. Agrawal AK, Singhal A, Gupta CM (1987) Functional drug targeting to erythrocytes in vivo using antibody bearing liposomes as drug vehicles, Biochem Biophys Res Commun 148(1): 357-361.

44. Chandra S, Agrawal AK, Gupta CM (1991) Chloroquine delivery to erythrocytes in Plasmodium berghei-infected mice using antibodybearing liposomes as drug vehicles. J Biosci 16(3): 137-144.

45. Urban P, Estelrich J, Adeva A, Cortés A, Fernàndez BX (2011) Study of the efficacy of antimalarial drugs delivered inside targeted immunoliposomal nanovectors. Nanoscale Res Lett 6: 620.

46. Drummond DC, Meyer O, Hong K, Kirpotin DB, Papahadjopoulos D (1999) Optimizing liposomes for delivery of chemotherapeutic agents to solid tumors. Pharmacol Rev 51(4): 691-743.

47. Stensrud G, Sande SA, Kristensen S, Smistad G (2000) Formulation and characterization of primaquine loaded liposomes prepared by a $\mathrm{pH}$ gradient using experimental design. Int J Pharm 198 (2): 213-228.
Creative Commons Attribution 4.0 International License

For possible submissions Click Here

\section{Submit Article}

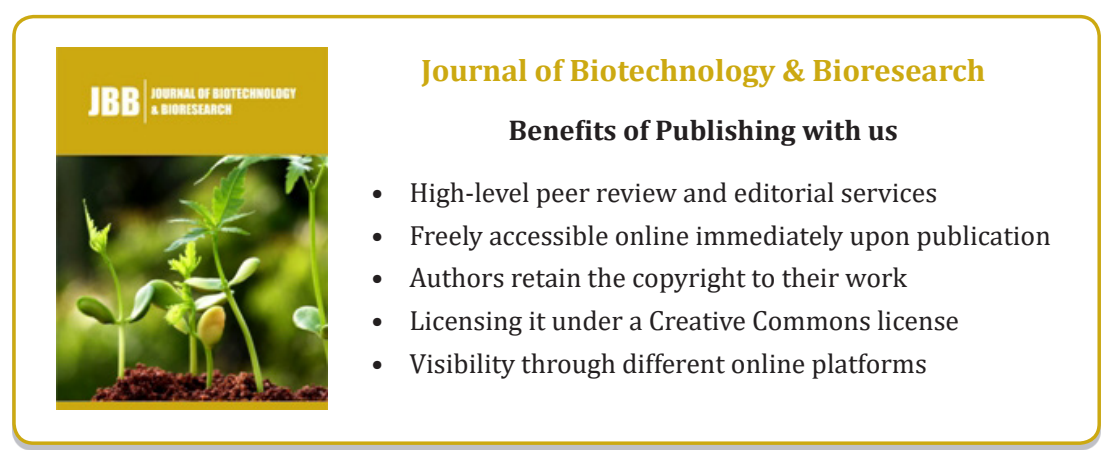

